\title{
PROYECTO ARQUITECTÓNICO EN LA REGENERACIÓN CULTURAL URBANA: EL CASTILLO DE LA LUZ EN LAS PALMAS DE GRAN CANARIA
}

\author{
Cesare Dallatomasina*
}

\section{RESUMEN}

Uno de los aspectos más debatidos en el ámbito arquitectónico de los últimos años es para qué y cómo recuperar un edificio histórico y devolverle protagonismo en cuanto testigo de la historia, incluyéndole su propia contemporaneidad, teniendo en cuenta su entorno e inserto en la ciudad. El presente trabajo trata del proyecto realizado por los arquitectos Fuensanta Nieto y Enrique Sobejano en el Castillo de La Luz de Las Palmas de Gran Canaria. Se ha considerado interesante analizar esta intervención por su capacidad de regeneración urbana de carácter cultural. Esta propuesta motivó un debate social que incluso propició una modificación proyectual y puso en primera línea informativa una cuestión de legado histórico. Actualmente es sede de la Fundación de Martín Chirino, aunando eficazmente una arquitectura recuperada y un nuevo uso, de ahí que se le pueda considerar un ejemplo representativo de recualificación.

PAlabras Claves: análisis, castillo, patrimonio arquitectónico, regeneración urbana, recualificación, reúso, uso cultural.

\section{ARCHITECTURAL PROJECT IN URBAN CULTURAL REGENERATION: THE CASTEL OF LIGHT (CASTILLO DE LA LUZ) IN LAS PALMAS DE GRAN CANARIA}

\section{Abstract}

In the last years, among the most debated approaches in field of architecture, there are the reason and the way to recover a historic building and return to prominence as a witness of history, including its own contemporaneity, moreover, considering its environment and its placement into the city. The present work deals with the project carried out by the architects Fuensanta Nieto and Enrique Sobejano of the Castle of Light in Las Palmas de Gran Canaria (Canary Islands, Spain). It has been interesting to analyse this intervention for the abilities in the urban regeneration of a cultural nature. This proposal generated a social debate that even led to a project modification and put in the front line of information a question of historical legacy. Currently, the Castle of Light is the headquarters of the Martín Chirino Foundation, effectively combining a recovered architecture and a new use, hence it can be considered a representative example of requalification.

KEYWORDs: analysis, castle, architectural heritage, urban regeneration, requalification, Canary Islands, reuse, cultural use. 


\section{INTRODUCCIÓN}

La arquitectura militar histórica perdió sus funciones originales al quedar obsoleta por los cambios operados en las cuestiones de defensa, de ahí que los derroteros de estos edificios fueron diversos al quedar abandonados y sin tener un uso específico. Si se repasara esta situación, incluso en países diferentes, se apreciaría que este patrimonio ha sufrido muchísimas e irreparables pérdidas, que han ido desde la desaparición de las murallas que poseían muchas ciudades hasta la demolición de castillos y otras dependencias militares, que en varios casos estaban en los centros urbanos. En la relación de los inmuebles conservados de este tipo se ofrece una variada situación, donde las intervenciones más afortunadas han logrado insertarlos en la contemporaneidad, devolviéndoles utilidad al servicio de la sociedad actual. En este trabajo se plantean algunas cuestiones relativas a un proyecto arquitectónico realizado en Gran Canaria en el paso del siglo xx al xxi, relevante en términos de restauración patrimonial. En el ámbito de Canarias, la situación en 1979 se resumía con "casos loables de conservación, como los lanzaroteños de San José (de la mano de César Manrique) o el de San Gabriel (Museo Arqueológico), ambos en Arrecife; el garachiquense de San Miguel; el de La Luz en Las Palmas y el caso de dudosa restauración científica de San Felipe del Puerto de la Cruz» ${ }^{1}$, a lo que se sumaba la denuncia del mal estado del Castillo de Santa Catalina de Santa Cruz de La Palma, del que se evidenciaba entonces su abandono por la «incultura y el progreso mal entendido que lo arrasan todo ${ }^{2}$.

Los ejemplos eran muchos más y desde entonces hasta el momento ha cambiado la situación, de tal manera que algunas de las intervenciones han servido no solo para la recuperación de este tipo de edificios, sino que por los nuevos usos han coadyuvado a dinamizar la ciudad contemporánea. En este trabajo se tratará el Castillo de La Luz, un ejemplo que después de pasar por diversas vicisitudes se ha integrado en la cotidianidad contemporánea de la ciudad de Las Palmas de Gran Canaria y en especial de los barrios de La Isleta y Puerto de La Luz, a partir del proyecto arquitectónico de Nieto y Sobejano (1998 y siguientes) y su conversión en sede de la Fundación de Arte y Pensamiento Martín Chirino (2015).

* Arquitecto por la Università di Parma (Italia). Doctorando de la Universidad de Las Palmas de Gran Canaria en el programa Islas Atlánticas: Historia, Patrimonio y Marco Jurídico Institucional.Email: cesaredallatomasina@gmail.com.

${ }^{1}$ López García, Juan Sebastián: «Defensa de la arquitectura militar», La Provincia, Las Palmas de Gran Canaria, 30 de noviembre de 1979.

2 Ibidem. 


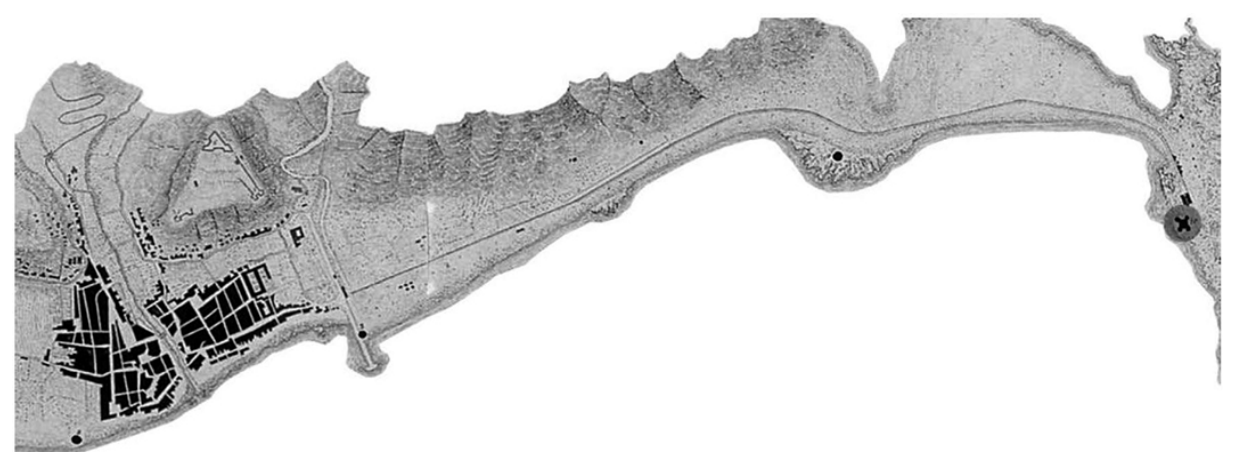

Fig. 1. Plano de la ciudad de Las Palmas, Nicolás Clavijo y Plo, 1857.

\section{CONOCER EL PASADO: SIGLOS XV-XX}

Como punto de partida para entender el presente del Castillo de La Luz es fundamental explicar las etapas que caracterizaron este proyecto, empezando por describir las fases iniciales de la fortaleza, declarada Bien de Interés Cultural en $1941^{3}$. Gracias al trabajo desarrollado por distintos investigadores ha sido posible recopilar material de documentación, exhaustivo hasta la mitad del siglo pasado, que de forma resumida hemos considerado interesante incorporar en este artículo para determinar de manera clara las principales modificaciones sufridas por el monumento. La intención no es generar un análisis nuevo de las etapas fundacionales y evolutivas de la fortaleza, sino demostrar la necesidad de explicar este proceso arquitectónico histórico para entender el proyecto de rehabilitación. Como resultado final hay que interpretar la restauración propuesta por Nieto y Sobejano, con las modificaciones aportadas inmediatamente después de la inauguración, convirtiendo el castillo en un nuevo centro cultural.

Los primeros testimonios de la existencia de un torreón probablemente de madera situado en el istmo de Las Isletas datan de 1480, época en la que Juan Rejón, tras su desembarco en 1478, estaba en plena conquista de Gran Canaria. La misión de este baluarte, y de todos los posteriores que se han sucedido en esta explanada, siempre ha sido proteger la bahía, en cuanto representaba uno de los puertos naturales de la isla por donde podían desembarcar los enemigos. Por esta razón en 1494 el tercer gobernador de Gran Canaria Alonso de Fajardo, hizo levantar una nueva torre, esta vez en piedra y cal, aprovechando posiblemente los cimientos de la de Juan Rejón. Como señala Pinto de la Rosa: «Ha sido D. Alonso de Fajardo, tercer Gobernador de Gran Canaria, en 1493 el promotor de la construcción de una fortificación donde surge el actual Castillo de la Luz. Sirvió como defensa a las muchas invasio-

\footnotetext{
${ }^{3}$ D. de 12 de julio de 1941. BOE 207, 26.07.1941.
} 
nes que sufrió la isla a finales del siglo xvi. A lo largo de los años ha ido ampliándose llegando a tener el volumen actual $»^{4}$.

Se trataba de una fortificación de planta cuadrangular de 10 metros de altura, ocho de lado y distribuida sobre tres niveles. El espacio de la cota cero estaba cubierto por un techo de bóveda de cañón; era la dependencia donde se encontraba la artillería pesada, que disparaba por unos huecos todavía visibles en las paredes. La primera planta tenía tres troneras de cerradura invertida orientadas a protección del eje este-sur y el acceso estaba garantizado a través de una escalera removible. El segundo nivel presentaba dos ventanas-troneras y comunicaba con la cubierta de la torre desde la cual a su vez se accedía a dos matacanes hoy desaparecidos. La imagen de la fortaleza se completaba con otros elementos, tal como se opina en la memoria que se redactó tras la excavación arqueológica del castillo (2005): «Es probable además que dicha cubierta estuviese provista de almenas y puede que incluso tuviera una cubierta de madera a modo de templete, para proteger los defensores. La cubierta fue arrasada cuando se acometen las reformas de 1572, quedando integrada en la explanada de artillería de la nueva fortaleza ${ }^{5}$.

Esta afirmación es muy útil para entender cómo se conformaban los principales volúmenes del castillo; además, hace referencia a la hipotética construcción de madera presente en la cima de la torre que, junto con las ampliaciones del siglo XVI, desapareció dejando espacio a una explanada, desarrollada en torno a un patio, donde quedó totalmente enterrado el antiguo baluarte. Por lo que, respecto al sistema defensivo alrededor de la atalaya y gracias al encuentro de las murallas durante las excavaciones realizadas para construir el actual centro cultural, fue posible verificar lo que testimonian varias crónicas y documentos de aquellos años, es decir, que fueron levantadas en distintas fases y una encima de la otra. Según estas fuentes documentales es posible comprobar que en torno al primer castillo se construyeron varias murallas, cada vez de mayores dimensiones, obteniendo siempre área fortificada más amplia.

En las primeras décadas del siglo Xvi el complejo defensivo que se configuraba no era suficiente para repeler todos los ataques que sufría la bahía de La Isleta; tanto que, después de varias invasiones y saqueos, en el año 1543 una tropa francesa consiguió apropiarse de la fortaleza durante dos días y una noche. Como consecuencia de esta vulnerabilidad, en el año 1548 el gobernador de Gran Canaria Ruiz de Miranda hace información pública sobre el estado del castillo pidiendo renovar la artillería y avances en las fortificaciones; un año después dicho informe fue enviado al rey. La petición tuvo éxito, ya que se adquirieron nuevos cañones y se realizaron ajustes en la muralla defensiva, de tal manera que «en 1549 el baluarte será sometido a una profunda remodelación, sobre todo de recrecido de los muros y posible-

${ }^{4}$ Pinto y de la Rosa, José María: Apuntes para la Historia de las Antiguas Fortificaciones de Canarias, Santa Cruz de Tenerife, 1996, p. 204.

5 Cuenca Sanabria, Julio; Guillén Medina, José y Tous Meliá, Juan: Arqueología de la Fortaleza de Las Isletas. La memoria del Patrimonio Edificado, Las Palmas de Gran Canaria, 2005, p. 63. 


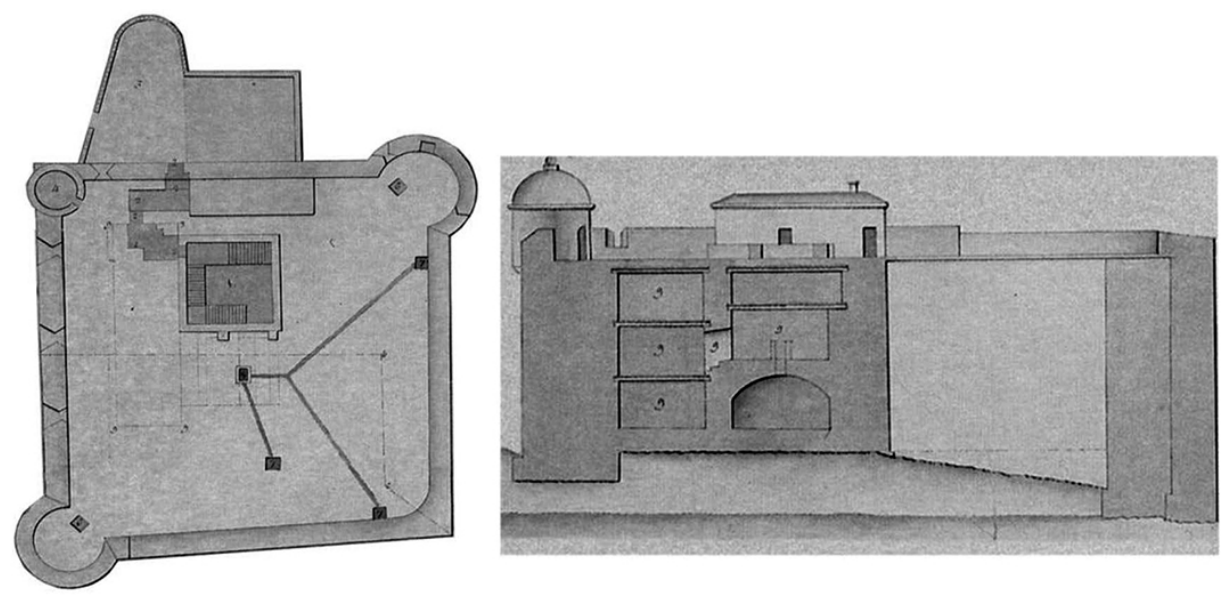

Fig. 2. Planta y sección de Castillo del Puerto de La Luz, Luis Marqueli y Bontempo, 1792.

mente a la apertura mayor de los huecos de las troneras, en los lienzos Este y Sur, con la finalidad de adecuarlo a las necesidades de las nuevas piezas de artillería ${ }^{6}$.

Desde el año 1553, gracias a las financiaciones particulares de Pedro Cerón y Ponce de León (1515-1574) ${ }^{7}$, fueron aportadas muchas mejoras al conjunto: se terraplenaron los muros perimetrales que miraban hacia el mar, se aumentó la altura de la barrera artillera y se construyeron las dos atalayas cilíndricas en las esquinas noreste y suroeste todavía existentes. Es en 1563, con el gobierno del licenciado Diego del Águila cuando empezaron los trabajos de modernización del complejo defensivo que conferirán a la fortaleza la forma que, excepto algunas modificaciones de bajo impacto en los siglos siguientes, llegará hasta mediados del siglo xx. En el año 1599 el castillo tuvo que afrontar una importante prueba contra la flota enemiga de los Países Bajos capitaneada por Van der Does y, a pesar de todas las reformas que se habían realizado, fue tomado durante algunos días y quemado una vez terminada la ocupación.

Los trabajos de reconstrucción empezaron inmediatamente después de la derrota y retirada de los holandeses, consiguiendo restablecer el anterior sistema defensivo en pocos años. Por estas fechas el puerto de Las Isletas adquiere el nombre de puerto de Nuestra Señora de La Luz, denominación otorgada por la presencia de la ermita de esa advocación. Durante los siglos siguientes y hasta la mitad del siglo XIX, el castillo continuó siendo pieza importante en el sistema defensivo de la

${ }^{6}$ Ibidem, p. 71.

7 Para este interesante personaje Vid. Caballero Mujica, Francisco: Pedro Cerón y el mayorazgo de Arucas, Arucas, 1973. 


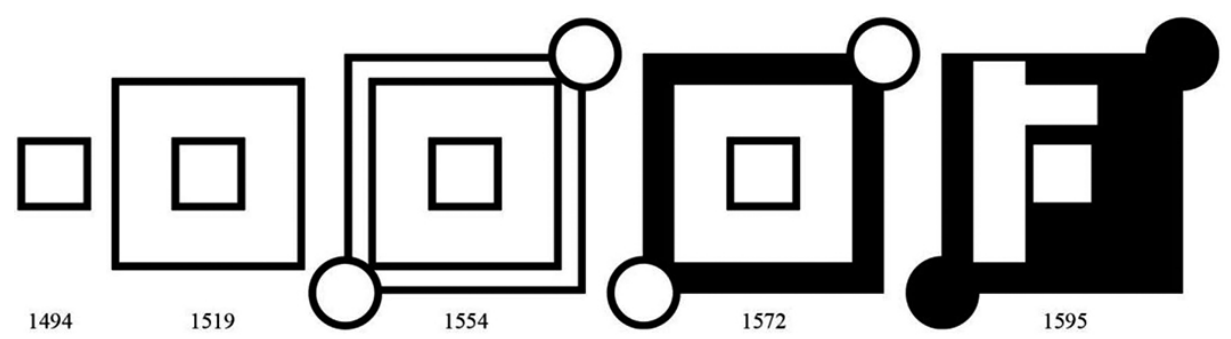

Fig. 3. Proceso evolutivo de las plantas bajas del Castillo de La Luz, siglos Xv-Xvi.

Esquema de elaboración propia.

isla ${ }^{8}$. En paralelo, la zona empezó a poblarse de forma constante y continuó su desarrollo económico, reflejado claramente en la expansión que llegó a tener en los siglos siguientes. A partir de la centuria decimonónica la fortaleza perdió gradualmente su función protectora, cayendo poco a poco en desuso hasta que en el año 1968 se propondrá un primer proyecto de recuperación con nuevo uso, aunque habrá que esperar hasta final del siglo xx para que se acometan las obras que permitirán recuperar íntegramente el inmueble.

\section{CONTEXTO URBANO, PROYECTO ARQUITECTÓNICO Y CAMBIO DE USO}

EL BARRIO DE LA ISLETA

Antes de analizar el proyecto de Nieto y Sobejano es oportuno examinar el contexto de la fortaleza para poder valorar las aportaciones de los arquitectos en el edificio y en el proceso urbano del sitio en el que se encuentra el castillo. Ubicado en la zona del puerto de La Luz de Las Palmas de Gran Canaria, cerca de los volcanes de La Isleta y, hasta hace pocas décadas, situado en una bahía natural hoy desaparecida. En este entorno se desarrolló un consistente núcleo habitacional formado por las originarias casas terreras que, poco a poco, dejaron espacio a bloques multiplantas modificando el paisaje del lugar. La vida y la economía de este barrio estuvieron estrechamente relacionadas con la pesca y con el comercio marítimo, actividades que se incrementaron en 1883 cuando la rada pasa de ser una bahía natural a una verdadera infraestructura portuaria, con las consecuencias que esto conlleva en términos de empuje económico y a la vez de problemas sociales y urbanos. Es sufi-

8 Tous Meliá, Juan: Gran Canaria a través de la Cartografía (1507-1899). Atlas históricogeográfico de la isla, San Cristóbal de la Laguna, 2014. 

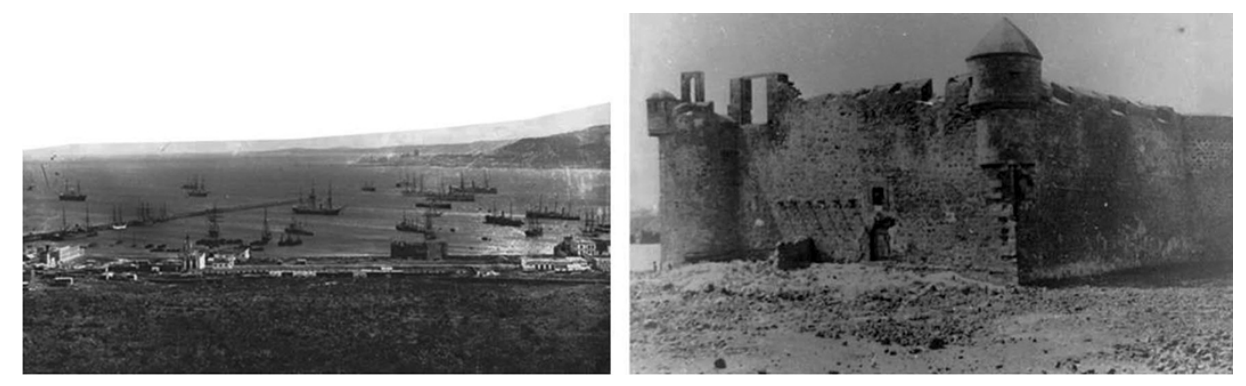

Fig. 4. Puerto de La Luz, 1895, y Castillo de La Luz, 1910. Fedac.

ciente pensar que en los últimos veinte años del siglo xix las laderas de los volcanes de La Isleta pasaron de alojar unas 100 personas a más de $11000^{9}$.

Es a partir de la segunda mitad del siglo xx cuando, gracias a factores como el turismo y nuevas actividades comerciales, empiezan unos lentos procesos de transformación de la zona. La presencia del Castillo de La Luz era el elemento de mayor prestigio del entorno, pero sin papel definido, a pesar de ser uno de los monumentos más importantes de la ciudad. La recuperación de la fortaleza y el establecimiento de la Fundación de Martín Chirino como nuevo centro cultural y de las iniciativas en él propuestas están sirviendo como elementos dinamizadores del barrio, jugando un papel fundamental en estos procesos de recuperación urbana y social. El conseguimiento de una renovada actividad del antiguo ente defensivo no ha sido fruto solo de las aportaciones de nueva planta avaladas por los arquitectos, sino también de la retirada de las mistificaciones «medievalistas» con las que se había adornado el castillo en las últimas décadas del siglo pasado. A raíz de esto cabe señalar que desde la segunda mitad del siglo xx las administraciones han tenido que dar respuestas a un paulatino boom turístico tanto en términos alojativos como culturales. Las reformas realizadas en 1969 fueron justo una consecuencia de esta necesidad de resanar rápidamente, y aparentemente de forma eficaz, un posible recurso turístico cultural.

Hoy en día el contexto donde se ubica la fortaleza está viviendo unos cambios significativos, pasando de ser un barrio poco atendido y fuertemente dependiente del factor portuario a un área conectada e insertada en el tejido urbano de la ciudad, con nuevas iniciativas económicas, culturales y turísticas. Una parte del mérito de esta regeneración hay que atribuirlo al crecimiento de las ofertas culturales propuestas por algunas entidades presentes en el distrito, como asociaciones de vecinos, talleres artísticos y centros de cultura. Algunas de estas transformaciones están ocurriendo gracias a la renovada centralidad del castillo y a los eventos

9 Galán, Fernando Martín: La formación de Las Palmas: ciudad y puerto. Cinco siglos de evolución, Las Palmas de Gran Canaria (1984), p. 211. 


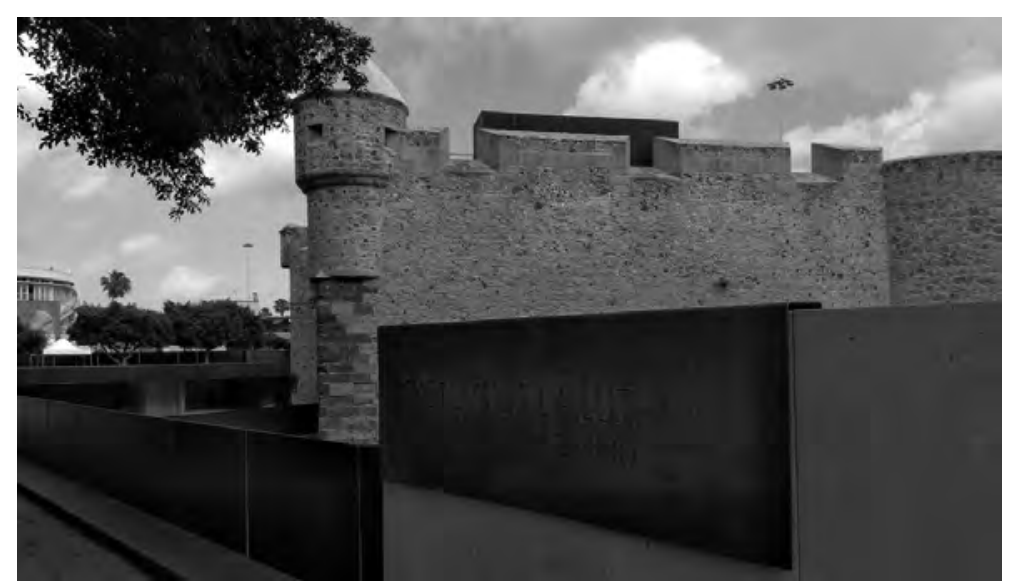

Fig. 5. Vista del Castillo de La Luz, sede de la Fundación de Arte y Pensamiento Martín Chirino, desde el parque exterior.

en él organizados. Estos procesos, unidos con los todavía palpables valores patrimoniales de carácter popular de La Isleta, están haciendo de este distrito un lugar particularmente dinámico ${ }^{10}$, un punto atractivo para canarios y extranjeros, con el ambiente de inclusión social que siempre ha caracterizado esta parte de la ciudad, y que desde antańo se evidenciaba con especial fuerza en los carnavales. Este concepto está evidenciado en la declaración de uno de los cofundadores de una asociación cultural ubicada en el barrio: «La esencia de una ciudad está en aquellos barrios que son pura autenticidad $»^{11}$.

\section{INTERVENCIONES ARQUITECTÓNICAS}

En algunos ámbitos el planteamiento proyectual de Nieto y Sobejano resultó algo atrevido en cuanto suponía modificar la fisonomía del castillo con algunos añadidos que especialmente afectaron a su perfil. Para entender bien el punto de partida de esta propuesta es determinante conocer el estado de la fortaleza antes de empezar las obras. Como es fácil imaginar, la situación del monumento era muy distinta a la que podemos ver hoy en día, manteniendo la herencia del siglo fundacional, cuando para mejorar las prestaciones bélicas del conjunto se optó por terraplenarlo dejando

${ }_{10}$ Uno de los espacios culturales que se han creado en esta zona es el Centro Cultural Pepe Dámaso, dependiente del Ayuntamiento de Las Palmas de Gran Canaria.

11 Vid: https://www.eldiario.es/canariasahora/sociedad/Isleta-centro-neuralgico-nuevastendencias_0_72 $\overline{6177883 . h t m l}$, consulta el 6 de marzo 2019, 13:00 horas. 
solo unos pocos vacíos en su interior. Con el pasar de los años esta estructura militar cayó poco a poco en desuso hasta que en la década de los sesenta del siglo pasado hubo un primer intento de recuperación transformando su sistema defensivo, típico de las Islas Canarias, dándole una imagen estereotipada más propia de un castillo medieval norteuropeo con foso y puente levadizo. En este confuso estado identitario, el monumento se demostraba capaz de acoger iniciativas de carácter cultural, como la muestra Héroes Atlánticos, que tuvo lugar en el año 1984 en conmemoración de la primera celebración del día de la Comunidad Autónoma de Canarias ${ }^{12}$.

Analizando las intervenciones de finales del siglo xx formuladas por Nieto y Sobejano, con el objetivo de devolver un aspecto de autenticidad a la obra, cabe señalar que los trabajos de restauración empezaron con la retirada de todos estos añadidos ajenos a la verdadera historia del monumento. En septiembre de 2001 comenzó una de las fases más delicadas de esta operación, que preveía el vaciado de la fortaleza para convertir los ambientes rellenados en las nuevas dependencias expositivas. Las obras se tuvieron que parar un solo mes después de su comienzo por la aparición de vestigios antiguos. La rápida intervención del Área de Patrimonio Histórico del Cabildo de Gran Canaria y de un equipo de arqueólogos logró dar luz a otros numerosos restos, ricos en informaciones históricas y documentales. Los importantes hallazgos desenterrados obligaron a cambiar el proyecto inicial adaptándolo a los nuevos testimonios encontrados. La relevancia de estos vestigios está bien descrita en el siguiente enunciado: «Durante los trabajos de restauración y rehabilitación del Castillo, un descubrimiento excepcional del que no existía ni la más mínima sospecha entre los especialistas que habían estudiado el pasado de este monumento. El hallazgo de las murallas primigenias del Castillo, posteriores a la torre de Alonso de Fajardo y anteriores a la edificación que conocíamos hasta hoy y de la que poseíamos representación gráfica a partir de los planos de L.Torriani, supuso un vuelco en el conocimiento histórico, arquitectónico, patrimonial y documental sobre la vida de este emblemático inmueble» ${ }^{13}$.

Examinando los pasos dados por los arquitectos en esta propuesta es evidente la voluntad de convertir el antiguo conjunto defensivo en un signo del presente, no obstante mantener su carácter de monumento y documento, intención que se refleja también en sus palabras: «La propia historia del Castillo de la Luz se convierte inevitablemente en argumento del proyecto $»^{14}$. Nieto y Sobejano, como demostración de querer transformar en realidad esta afirmación, ya desde el principio de sus intervenciones, vaciaron los interiores de la fortaleza reportando a la luz muchos espacios desaparecidos y convirtiéndolos en volúmenes fundamentales del nuevo conjunto. Los trabajos fueron complejos y con herramientas que permitieron recuperar los vestigios de los niveles más bajos: «Durante esta fase de las obras

${ }^{12}$ Con tal motivo se organizó una exposición del artista José Dámaso, Vid. AA. VV.: Héroes Atlánticos. Pepe Dámaso, Las Palmas de Gran Canaria, 1984.

13 Cuenca Sanabria, Julio, Guillén Medina, José y Tous Meliá, Juan: op. cit., p. 11.

${ }^{14}$ Nieto, Fuensanta y Sobejano, Enrique: op. cit., p. 148. 
se emplearía maquinaria pesada, por lo que en poco tiempo se pudo alcanzar una gran profundidad, hasta que afloraron los restos de una muralla interior construida a base de piedra con mortero de cal, que parecía presentar cuatro lienzos que discurrían en paralelo con la muralla exterior ${ }^{15}$. Como consecuencia de la afloración de estos inesperados hallazgos arqueológicos los arquitectos, cambiando el originario proyecto, optaron por integrar los restos de la muralla a la sala principal tratándolos a la misma vez como obras de arte y testigos evolutivos del castillo. Para establecer una jerarquía entre las partes más antiguas, muchas encontradas durante las excavaciones arqueológicas, y las más recientes, Nieto y Sobejano realizaron una diferenciación cronológica de los varios elementos utilizando distintos revestimientos. Por lo que concierne a las superficies del núcleo fundacional de la fortaleza, identificado en la torre central y su primera fase, fueron tratadas manteniendo las conformaciones y texturas originales, mientras las partes que se construyeron inicialmente para estar en contacto perenne con la tierra, como por ejemplo las caras interiores de los muros perimetrales, se pintaron de blanco. El empleo de este color no es casual, la elección de esta tonalidad sirve para responder ideológicamente a esta pregunta: si nadie podía ver las texturas de estas paredes, ¿por qué tenemos que verlas nosotros? ${ }^{31}$. Con esta estrategia se consiguió distinguir los elementos antiguos de los actuales y, estableciendo una jerarquía cronológica, se facilitó su lectura.

Otro aspecto que merece ser resaltado es la elección de los materiales que los arquitectos han escogido para las nuevas inserciones. En el ascensor, las vallas de las escaleras y las pasarelas han optado por el acero corten, en la cubierta se ha empleado el hormigón armado dejado en su estado bruto, mientras en el techo se colocaron unos paneles de vidrio que, rodeando la torre y los muros perimetrales, permiten a la luz entrar y resbalar en las paredes enfatizando sus texturas. El espacio expositivo se encuentra en los volúmenes obtenidos por las excavaciones y en los pocos vacíos que ya existían, se distribuye en tres distintas cotas alcanzables con recorridos que conectan todas las partes de la fortaleza y que atraviesan también los dos cubelos circulares. Las cubiertas, tanto la del castillo como la del nuevo edificio exterior, no son transitables al público. Una de las aportaciones que han conferido más identificabilidad a este proyecto ha sido la disposición de una ventana rectangular de acero corten colocada en la fachada de acceso al conjunto defensivo, inserción que no es un formalismo arquitectónico, sino una "vera» reconstrucción de lo que era en origen este elemento: una tronera de piedra colapsada en el siglo pasado. La existencia de documentación fotográfica ha permitido una reconstrucción igual en la forma pero renovada en el material empleado. Esta abertura tiene la misma composición que las que se encuentran en el interior de la fortaleza, todas igualmente enmarcadas por el mismo metal. Este moderno mirador representa una excepción que dife-

15 Cuenca Sanabria, Julio, Guillén Medina, José y Tous Meliá, Juan: op. cit., p. 30.

16 Conferencia de Fuensanta Nieto con título «Denkbilder» en el ciclo de la Semana de la Arquitectura 2017, Fundación de Arte y Pensamiento Martín Chirino, Las Palmas de Gran Canaria, 5 de octubre 2017. 


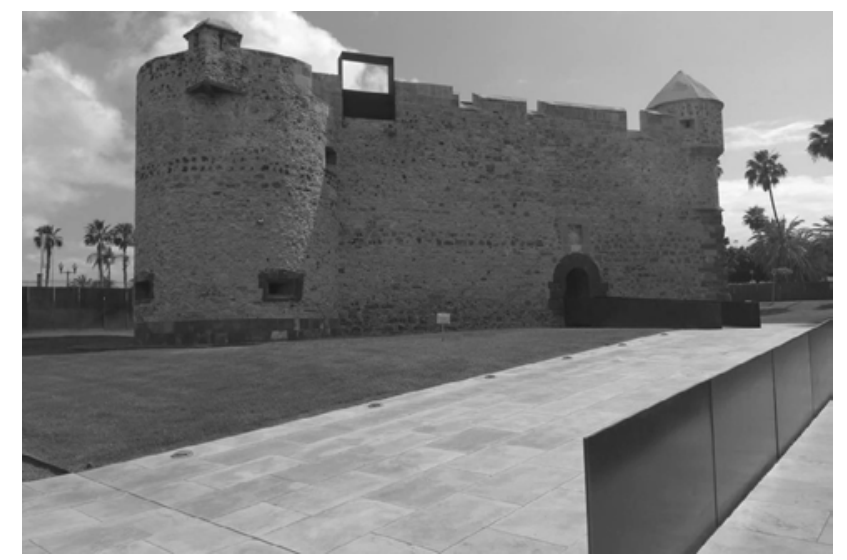

Fig. 6. Castillo de La Luz con algunos de los insertos en acero corten: ventanal-mirador y pasarelas.

rencia este hueco de las otras ventanas, es el único de estos recuadros que no está rodeado por piedra, caso singular de nuevo sin lo viejo. Con la solución que acabamos de examinar los arquitectos han demostrado la posibilidad de restablecer algo antiguo desaparecido a través una estrategia contemporánea, haciendo apropiada la siguiente frase de Antoni Gaudí: «Hagamos arquitectura sin arqueología: ante todo están las relaciones entre las cosas, en una situación predispuesta; por esto no debemos copiar las formas, sino estar en condición de producirlas dentro de un determinado carácter, poseyendo su espíritu $»^{17}$.

Aprovechando las operaciones de remoción de los añadidos de estilo medieval, el área del foso se dotó con césped, estableciendo así un entorno de apariencia natural capaz de evocar su originario ambiente caracterizado por rocas y agua. En un lado de esta explanada se dispuso la única estructura de reciente construcción antecedida por un espacio pavimentado al aire libre con función de plaza y de filtro entre el nuevo edificio y el castillo. Este pequeño edificio ha sido insertado con discreción en este entorno patrimonial, revelándose capaz de dialogar con la fortaleza sin entrar nunca en conflicto con ella. Se trata de una construcción rectangular que casi desaparece en la pendiente natural del terreno; en su interior se encuentran todos los servicios de los que mínimamente necesita un museo y que, por razones de espacio, no se podían disponer dentro del monumento: venta de entradas, publicaciones, aseos, almacén, instalaciones y sala polivalente. Los materiales escogidos para este nuevo edificio son el vidrio y el hormigón bruto para las superficies verticales y el techo, una piedra de color claro en el suelo de tránsito mientras en la cubierta

${ }_{17}$ González Moreno Navarro, Antoni: La restauración objetiva: (Método SCCM de restauración monumental) Memoria SPAL 1993-1998, Barcelona, 2000, p. 63. 
se utiliza el elemento que más caracteriza este planteamiento, es decir, el acero corten, que casualmente tiene mucho que ver con el artista al que se dedicará el espacio expositivo, ya que Chirino ha destacado en su escultura por el magistral uso de la forja. Toda el área del conjunto está delimitada por una valla de este mismo metal con el objetivo de crear un ambiente renovado y al mismo tiempo capaz de enlazar con el que tuvo el castillo hasta la mitad del siglo pasado. Con esta solución los arquitectos han restablecido la cota original de la época fundacional del antiguo conjunto defensivo consiguiendo resolver el desnivel del terreno con una pasarela que pone en comunicación el barrio con el nuevo centro cultural; dicho puente de entrada desemboca en la plaza descrita previamente conformando en determinadas ocasiones un verdadero foyer a cielo abierto.

Un aspecto bastante debatido de este planteamiento fue la altura de la valla perimetral en acero corten que delimita el conjunto. Hasta el año 2015 tenía un tamaño tal que casi no permitía admirar el castillo desde el jardín y a la misma vez no dejaba ver el barrio desde el interior del centro cultural. La voluntad de los arquitectos era justo esa, no abrir la fortaleza a la ciudad por los muchos cambios que sufrió, sobre todo en las últimas décadas, evitando así instaurar un difícil diálogo con las partes que se han producido en su alrededor. Uno de los fines de esa inserción era extrapolar el monumento de la heterogénea conformación urbana que lo rodea, caracterizada por la presencia de vías de tráfico rodado y autovías, edificios de grandes alturas, naves industriales que fueron soporte de la economía portuaria, supermercados y un jardín a la francesa extraño al litoral que antiguamente acogía el castillo. Con esta solución se concibió un ambiente cerrado donde el visitante podía pasar de un contexto urbano y caótico a uno natural y aséptico en el que era palpable esta relación entre historia y contemporaneidad. El resultado fue la creación de un contexto nuevo e introspectivo en el que el huésped se pudiera sentir como aquellos usuarios del siglo Xvi contornados solo por el monumento y la naturaleza. Una vez inaugurado el nuevo proyecto los debates surgidos y las opiniones contrarias motivaron la decisión de la administración de cortar la valla confiriendo al conjunto el aspecto que podemos ver hoy en día.

Finalmente, después de todo este debate, la Fundación de Arte y Pensamiento Martín Chirino se instaló dentro de un jardín público existente y, analizándola desde una perspectiva más amplia, es fácil notar cómo la intención de Nieto y Sobejano era la de instaurar un espacio diferente al del castillo, de uso comunitario y destinado a una función distinta. El objetivo era separar lo lúdico de lo cultural reservando un área exclusivamente para la historia y el patrimonio con la obra de Chirino entendida como legado, y asignando a la otra los eventos y actividades creativas. En este sentido cabe señalar las muchas iniciativas de carácter cultural que tienen lugar en la plaza, en el parque y en el auditorio de la fundación: actos musicales de varios géneros, la semana de la arquitectura, congresos, conferencias, etc. Dicho y aceptado esto, es posible entender como legítimas las protestas de una parte de los habitantes de La Isleta, sobre todo de los que nacieron y se criaron en el barrio, acostumbrados a la presencia de la fortaleza tanto en términos visuales como de accesibilidad. Las quejas principales estaban dirigidas hacia la altura de la valla y a la decisión de crear un ambiente con entradas restringidas en cuanto con- 


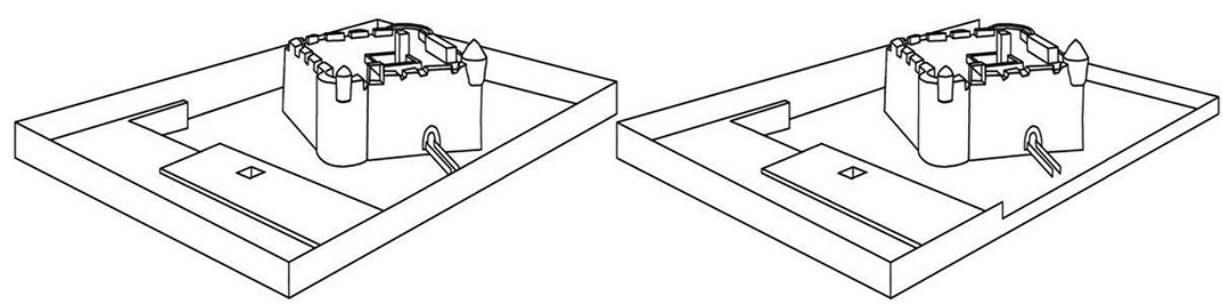

Fig. 7. Corte de la valla perimetral del Castillo de La Luz, mayo 2015.

Esquema de elaboración propia.

cedido a una fundación privada. En el fondo hay que preguntarse a quién pertenece el castillo, si a los que lo han construido y lo han utilizado en su originaria función hace aproximadamente 500 años, a aquellos que lo han visto integrarse en el actual tejido urbano convirtiéndose poco a poco en uno de los símbolos de la ciudad, o a las generaciones futuras y entonces a la modernidad. En estos contextos patrimoniales la arquitectura tiene una función de costura entre todas las fases que el bien ha atravesado, sin olvidarse de ninguna. Este posiblemente es el aspecto más complicado de esta disciplina por su pertenencia a distintos momentos de la historia.

\section{NACimiento DE Un NUEVo CENTRO CULTURAL}

La Fundación de Arte y Pensamiento Martín Chirino, tras su creación, se instaló definitivamente en el Castillo de La Luz, afectando y "contaminando" de manera propositiva su entorno. Una vez perdido su uso militar y sin utilidad específica para la ciudad, el Ayuntamiento de Las Palmas de Gran Canaria, con la colaboración de fondos europeos, promocionó la convocatoria de un concurso de proyectos arquitectónicos con el objetivo de restaurar la fortaleza. En el año 1998 el Área de Patrimonio Histórico del Cabildo aprobó la propuesta avalada por los arquitectos Fuensanta Nieto y Enrique Sobejano, como ya se ha expuesto. En un primer momento la comisión pública había decidido convertir el monumento en Museo del Mar, planteando entonces los arquitectos todas las dificultades típicas que se encuentran en la conversión de un espacio antiguo y en estado ruinoso en un edificio útil con funciones nuevas, contemporáneas y capaz de adaptarse a eventos de varios tipos. Durante los ańos de las obras los usos previstos fueron distintos y al final las opiniones de las administraciones públicas coincidieron en dedicar este espacio al escultor Martín Chirino ${ }^{18}$. Esta decisión supuso que el 28 de marzo de 2015 este renovado complejo arquitectónico, provisto de auditorio, espacios expo-

18 Martín Chirino López (Las Palmas de Gran Canaria, 1 de marzo 1925-Madrid, 11 de marzo 2019). 


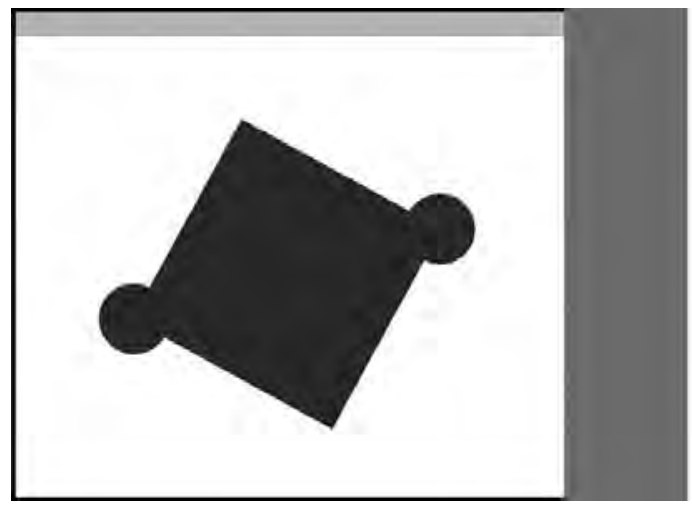

Pasarela de acceso

Nuevo edificio

Castillo

Valla perimetral

Fig. 8. Esquema general del conjunto. Elaboración propia.

sitivos y de todos los locales que conciernen a este tipo de infraestructuras, se inaugurara como la Fundación de Arte y Pensamiento Martín Chirino. La asignación de la fortaleza a este último y todavía actual destino generó no pocas polémicas en cuanto algunos habitantes querían un museo que contara la historia de La Isleta y del puerto, un lugar que fuera público y apto a las iniciativas populares del barrio y no un sitio que consideraban privado y casi elitista.

No es intención de este trabajo pronunciarse de manera favorable o contraria al destino escogido para este monumento, ya que nuestro propósito es estudiar el conjunto bajo una mirada arquitectónica y urbana. En este sentido cabe señalar que la entidad de un ambiente como este se puede adaptar a las diferentes funciones, cumpliendo en cada caso con las expectativas, ya que se está frente a un proyecto respetuoso con el pasado y al mismo tiempo emblema de modernidad, capaz de combinar lo antiguo con lo nuevo. Este tipo de edificios solo hay que ponerlos en las mejores condiciones para que sean representantes de sus historias. Una de las mejores maneras para entender el proyecto de Nieto y Sobejando es la de leer sus ideas especialmente expresadas para esta restauración: «Más que reconstruir o rehabilitar el castillo, lo habremos vaciado, nos habremos limitado a hacer visible su pasado esperando que el edificio, independientemente de las futuras colecciones que a él se incorporen, se exponga ante todo a sí mismo y a su propia historia» ${ }^{19}$.

19 Nieto, Fuensanta y Sobejano, Enrique: Desplazamientos. Displacements, Madrid, 2002, p. 148. 


\section{ANÁLISIS DEL PROYECTO ARQUITECTÓNICO DE FUENSANTA NIETO Y ENRIQUE SOBEJANO}

La considerable cantidad de material gráfico publicado sobre el proyecto de intervención del Castillo de La Luz realizado por Nieto y Sobejano contrasta con la poca información literaria, que se reduce casi exclusivamente a algunos escritos de los autores. Dicha escasez documental dificultó bastante el análisis y entendimiento de muchas cuestiones acerca de las decisiones proyectuales tomadas por los arquitectos, carentes de explicaciones directas por los mismos. En este trabajo de investigación ha sido necesario un esfuerzo de continua comparación entre el proyecto actual y las cartografías antiguas para poder apreciar los cambios y entender las decisiones escogidas por los restauradores. En cada caso se ha comprobado el conocimiento y la consideración de la historia de la fortaleza y la capacidad de adaptar un bien antiguo a funciones culturales contemporáneas, precisado de ser dotado de todas las instalaciones requeridas para que un espacio como este pueda funcionar. La elección de los materiales, los volúmenes, las funciones, etc., en ningún momento han seguido meros caminos estéticos o ligados al gusto personal, han sido siempre fruto del estudio del monumento y del lugar. En esta, como en otras obras, el dominio del pasado tendría que ser el requisito mínimo para cualquier especialista intencionado a operar en contextos patrimoniales.

Desde finales siglo xx y principios del xxi el Castillo de La Luz ha sido foco de muchos debates y polémicas; en este capítulo se analizan los procesos evolutivos que mayormente han caracterizado este arco temporal. Al mismo tiempo, se han querido extraer las soluciones arquitectónicas adoptadas con el fin de dar a conocer esos planteamientos por si puedan servir de referencia para futuras puestas en valor del rico patrimonio arquitectónico que nos rodea. Vivimos en un archipiélago que ha perdido gran parte del legado de sus sistemas defensivos que tanto han significado a lo largo de su historia y desde la arquitectura es tarea primordial proponer la recuperación de los pocos monumentos de este tipo que se han conservado, algunos en estado ruinoso o abandonados y otros necesitados con urgencia de una recualificación capaz de devolverlos para ser usados por sus ciudades y sus habitantes. El Castillo de La Luz es la demostración de cómo una buena recuperación patrimonial puede ser doblemente beneficiosa, ya que ha permitido reconquistar un bien abandonado y dinamizar su entorno inmediato. El análisis realizado en este artículo nos permite identificar cuáles han sido las principales intervenciones que caracterizaron el proyecto de Nieto y Sobejano, dando a esta investigación el mérito de ir más allá de una simple enumeración cronológica de las etapas fundacionales de la obra y de su restauración, ya que se ha considerado de relevante interés estudiar críticamente las propuestas de los arquitectos de manera que puedan servir como punto de partida para otros planteamientos con similares características. Las decisiones tomadas en este proyecto de restauración reflejan el sentido común de los diseñadores y, analizándolas de forma teórica extrapolándolas de un plano de dibujo o de la realidad, demuestran ante todo que son compartibles. Aspectos tales como la colaboración de distintos especialistas, defensa del contexto del monumento, diferenciación e identificación entre moderno y antiguo (que estos sean nuevos volúmenes o 


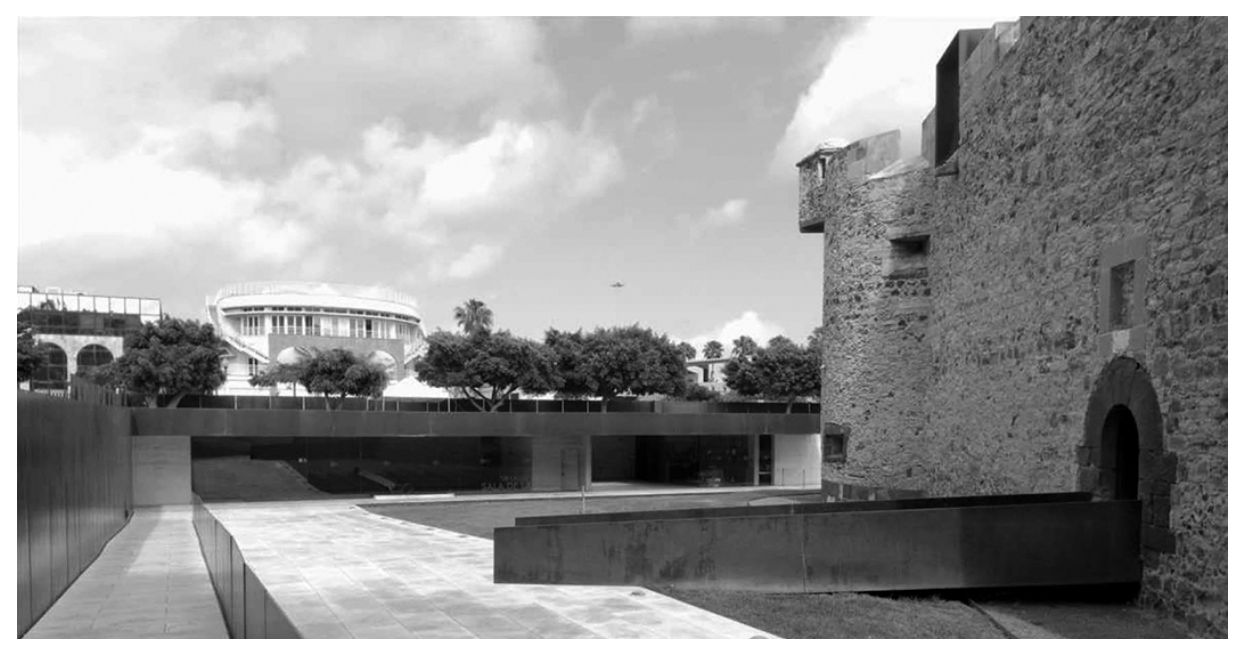

Fig. 9. Puentes de entrada a la Fundación de Arte y Pensamiento Martín Chirino y al Castillo de La Luz.

reconstrucciones), elección mínima de materiales empleados de manera que no se quite protagonismo a los originales del hallazgo, inserción de las nuevas construcciones de forma respetuosa y mimética representan solo algunas de las principales aportaciones proyectuales y sin duda aplicables a la mayoría de cualquier proyecto de recualificación patrimonial.

\section{EQUIPO INTERDISCIPLINAR}

La confrontación entre distintas disciplinas siempre ayuda al debate consiguiendo alcanzar un resultado mejor y con mayor certeza, colaboración interprofesional que tendría que ser exigida en la mayoría de las intervenciones patrimoniales. Un caso lo tenemos en el castillo: para la recuperación de este monumento fue de fundamental eficacia el que dispusiera casi desde el principio de un equipo interdisciplinar. De vital importancia ha sido la presencia de los arqueólogos, que han descubierto una considerable cantidad de material, integrado con acierto al conjunto gracias al trabajo de los arquitectos. Una demostración clara fue que en los primeros meses de excavación, cuando todavía no se había incorporado el equipo de arqueólogos al grupo de trabajo, se perdió una gran cantidad de material probablemente rico en informaciones, a causa de haber empleado maquinarias pesadas durante el proceso de vaciado. Hay que decir también que sin el empleo de estas maquinarias se hubiera llegado a la terminación del proyecto en mucho más tiempo, con repercusiones significativas en la duración de la obra y en el coste total 
de la intervención. De todas formas cabe señalar que los restos de mayor prestigio como el torreón central con su primitiva muralla, se salvaron, posteriormente se restauraron, y fueron justo estos descubrimientos los que determinaron la entrada del equipo arqueológico.

Nieto y Sobejano en esta fase inicial prefirieron primar los restos con valores arquitectónicos en detrimento de otros ajenos a lo constructivo, decisión que hizo perder mucha información, porque, en otras palabras, la tierra extraída seguramente contenía vestigios de pequeñas dimensiones con datos interesantes como la manera de vivir de los usuarios del castillo, pero se priorizaron solo los hallazgos que eran capaces de contar la historia del edificio, considerando estos como los testigos de mayor interés. Uno de los objetivos de los proyectistas fue devolver el monumento en el menor tiempo posible a la colectividad, determinación que en su elección es típica de un pensamiento arquitectónico que encuentra paralelo en lo que dijo Francesco Venezia en referencia a un caso parecido en Nápoles: «In questo momento a Napoli piazza Municipio è da circa dieci anni una fossa enorme. (...) Nella quale indomiti archeologi con le loro scopette stanno ripulendo fra l'altro le fondazioni dei bastioni demoliti negli anni trenta -ottant'anni fa!- paralizzando la realizzazione di una stazione della metropolitana progettata da un bravo architetto che rischia di non vedere il suo progetto realizzato, e con lui un'intera generazione è privata del godimento di una piazza centrale della propria città $»^{20}$.

Lo ideal, y creemos que no sea una visión utópica, sería que arqueólogos y arquitectos encontraran la manera de colaborar buscando un punto común proponiendo intervenciones que no comprometan los valores documentales y a la vez permitan convertir el bien en algo utilizable en tiempos razonables. Los monumentos pertenecen a los ciudadanos, existen para ser vividos y no puestos dentro una vitrina para ser contemplados, lo que no significa que se puede hacer con ellos lo que se quiere, ya que siempre tendrán que transmitir su historia y mantener sus valores documentales. En este sentido es útil mencionar la frase expresada por el ingeniero y arquitecto Marco Dezzi Bardeschi: «Ridefiniamo quindi il restauro come la sommatoria di due ordini di operazioni: Restauro = progetto di conservazione dell'esistente (come valore complessivo) + progetto del nuovo (come valore aggiuntivo) $)^{21}$.

${ }^{20}$ Venezia, Francesco: Che cos'è l'architettura - Lezioni, conferenze, un intervento, 2011, p. 17. «En este momento Piazza Municipio de Nápoles, desde hace aproximativamente 10 años, es una enorme fosa. [...] En la que indómitos arqueólogos con sus pinceles están limpiando, entre otras cosas, los cimientos de los bastiones demolidos en los años treinta - ¡hace ochenta ańos!- paralizando la construcción de una estación de metro diseñada por un buen arquitecto que corre el riesgo de no ver nunca su proyecto realizado, y con él toda una generación se ve privada del disfrute de una plaza central de su ciudad». Traducido por el autor.

${ }^{21}$ Venturini, Simone: Il restauro cinematografico: principi, teorie, metodi, 2006, p. 44. «Redefinimos entonces la restauración como la suma de dos órdenes de operaciones: Restauración $=$ proyecto de conservación de lo existente (entendido como valor global) + proyecto de lo nuevo (entendido como valor adicional)». Traducido por el autor. 

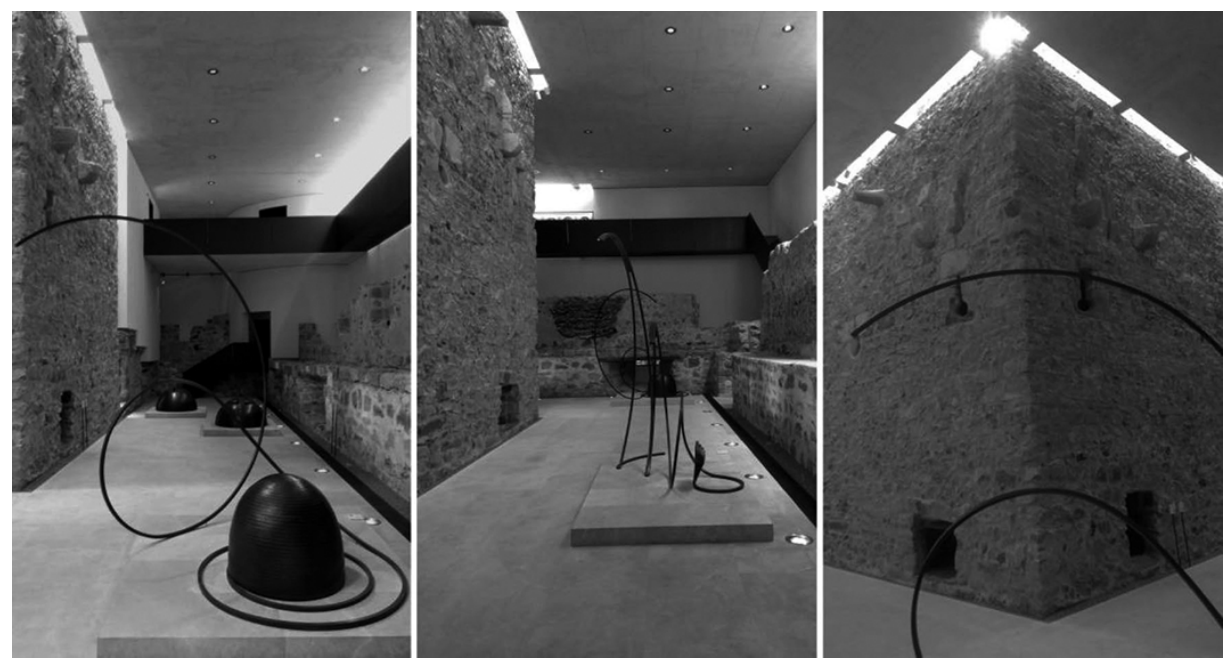

Fig. 10. Vistas de la sala expositiva desarrollada alrededor de la torre erigida por Alonso de Fajardo en 1494.

\section{DeFENSA DEL CONTEXTO ORIGINARIO DEL MONUMENTO}

En esta fase del trabajo se quiere subrayar la importancia de defender y poner en valor el contexto de un monumento, es decir, el ambiente urbano en el que se encuentra un determinado bien patrimonial, sea este todavía el originario, fruto de transformaciones o totalmente desaparecido. Continuando con el análisis del proyecto del Castillo de La Luz examinaremos ahora una parte de la intervención que hoy es solo parcialmente identificable en cuanto una vez terminadas las obras, a pocos meses de la inauguración, se decidió modificarla. Nos referimos a la valla perimetral, barrera de acero corten con la que se rodeó el edificio, que actuó como un muro y se diseñó sin considerar las consecuencias que suponía en términos sociales. Sin embargo, cabe señalar que los arquitectos con esta idea querían crear un ambiente nuevo y que nunca había existido alrededor del monumento, capaz de evocar el antiguo desaparecido y resultando contemporáneo a la vez. La valla fue recortada, aunque, posiblemente la propuesta era novedosa y el propósito de establecer un renovado envolvente a la obra se produjo privilegiando los aspectos paisajísticos (naturales y arquitectónicos) a costa de los valores humanos y sociales del barrio con consecuentes, y comprensibles, polémicas por parte de los ciudadanos. Cada porción urbana está formada ante de todo por las personas que la viven. Como dijo Antoni González Moreno Navarro: «Restaurar no es sólo rehacer el monumento (es decir, volver a hacer lo deshecho), sino recuperar sus significa- 
dos, reinterpretar su pasado para el presente y para el futuro $»^{22}$. Esta solución arquitectónica, si es empleada con una mayor consideración de los que verdaderamente poseen y utilizan el bien, es decir, los ciudadanos, podría ser una válida opción para futuras restauraciones. Varios monumentos necesitan ser recualificados justo en las que son las relaciones entre los contextos patrimoniales y las nuevas conformaciones urbanas que se han producido en sus alrededores, por esta razón hemos considerado interesante destacar esta actuación que trajo debate y cambio del proyecto.

INTEGRACIÓN DE LOS RESTOS ARQUEOLÓGICOS Y NUEVOS VOLÚMENES EN EL PROYECTO ARQUITECTÓNICO

Otro aspecto que merece particular atención es lo relativo a la capacidad de adaptabilidad del proyecto a los hallazgos encontrados. Un planteamiento arquitectónico que incluya excavaciones arqueológicas de esta relevancia tendrá que afrontar muchos problemas y cambios imprevistos, demostrando su elasticidad a los valores patrimoniales encontrados. Los diseñadores del caso de estudio aquí examinado evidencian sus habilidades en la puesta en valor de los elementos desenterrados, integrándolos al proyecto y dando uniformidad al conjunto. Una situación digna de ser mencionada y tomada en consideración en el futuro se refiere al tratamiento que Nieto y Sobejano reservaron para la muralla del primitivo torreón, aparecida durante las operaciones de vaciado y resuelta con la colocación de las salas expositivas principales a lo largo de su longitud, considerándola entonces como una verdadera obra de arte que se puede admirar caminando a su alrededor. Gracias a las diferenciaciones de los materiales, los sistemas de entrada de la luz cenital y la disposición de los ambientes dedicados a las muestras, han conseguido dar la impresión de que estos restos pertenecían ya a la idea inicial del planteamiento, como si casi se supiera previamente de su existencia.

El conjunto está compuesto también, como ya se comentó, por un edificio de nueva construcción; en este caso los arquitectos se han mostrado respetuosos con las prexistencias monumentales, planteando un espacio parcialmente «hipogeo», insertado en la pendiente del terreno para mitigar el impacto de su volumen y que afectara lo menor posible al debido protagonismo de la fortaleza. La intención era justo la de no crear contrastes, se erigió un ámbito necesario para poder desenvolver completamente las funciones del centro cultural, afirmando claramente su carácter «secundario» que representa un ambiente de soporte meramente funcional del monumento. Este deseo está enfatizado por la elección de materiales de tonalidades neutras y de bajo impacto visual, por el cierre frontal en vidrio que confiere una sugestiva transparencia al conjunto y por una estructura perimetral formada por tres paredes de hormigón que definen la planimetría y al mismo tiempo sirven

22 González Moreno Navarro, Antoni: «Construir el pasado», Informes de construcción, vol. 45, n. ${ }^{\circ}$ 427, (1993), sin página. 


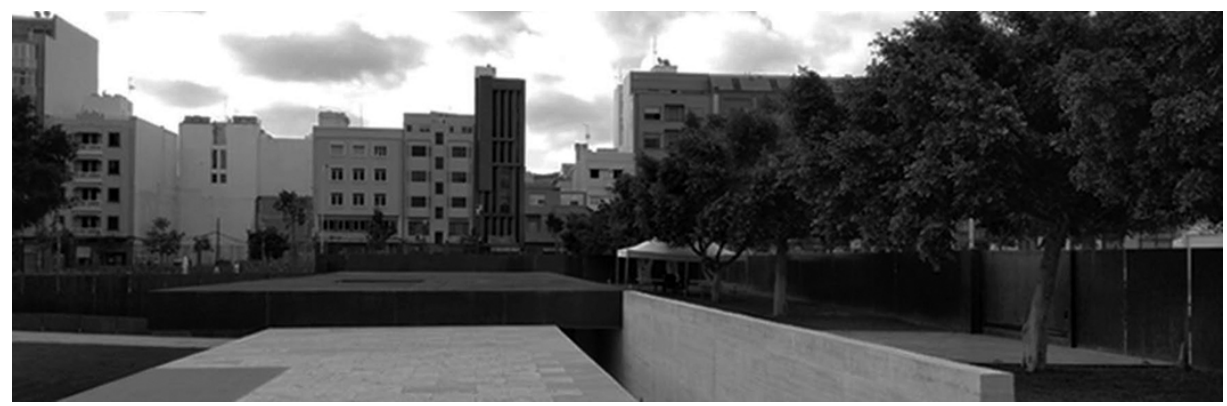

Fig. 11. Enfoque fotográfico de cómo los arquitectos han conseguido insertar un jardín privado y exclusivo para la Fundación de Arte y Pensamiento Martín Chirino entre el edificio de nueva planta y la valla externa.

como muros de contención para la instalación de un jardín situado a la misma cota del parque exterior al centro cultural. Este rectángulo verde más elevado se abre al público solo en determinadas ocasiones, normalmente en eventos que requieren un espacio al aire libre donde sea igualmente garantizada una cierta intimidad. Gracias a este sistema de soporte de tierra ha sido posible conformar un área natural privada y escondida entre el techo de este nuevo edificio y la valla perimetral del conjunto.

\section{CONCLUSIONES}

Con este trabajo se ha querido sintetizar y agrupar en una única recopilación los procesos constructivos del Castillo de La Luz con un breve análisis, desde sus orígenes en el año 1480 hasta las intervenciones y cambio de uso del siglo XXI. Para entender las reformas aportadas por Nieto y Sobejano, y en general de cualquier otro proyecto de restauración arquitectónica, es fundamental conocer el pasado del bien objeto de estudio. Durante los últimos años de las obras y también después de la inauguración del nuevo centro cultural, el Castillo de La Luz ha sido objeto de polémicas, algunas parcialmente compartibles con lo defendido en este trabajo, y otras, fruto de simples y meros juicios estéticos carentes de fundamentos cognitivos sobre el edificio. Estas críticas han ayudado a reflexionar sobre las soluciones que aportaban los autores del proyecto junto con las de contenido contrario. De cualquier manera, para tomar una decisión lo más acertada posible en este debate, ha sido fundamental el conocimiento, aunque sea escueto, de la historia de la fortaleza y así entender las transformaciones del planteamiento de Nieto y Sobejano, consiguiendo también dar respuesta a algunas críticas.

Entre las soluciones que fueron cuestionadas, la creación de un espacio interior de muros opacos, el empleo del acero corten como material caracterizador, las diferenciaciones de tratamiento de los paramentos en los espacios expositivos y la 
estrategia con la que se han insertado los nuevos volúmenes reflejan solo una parte de los aspectos más criticados de este proyecto. Estas son solo algunas de las aportaciones tratadas y estudiadas, gracias a las cuales fue además posible confeccionar una serie de posibilidades a la que poder acceder en caso de futuros proyectos de puestas en valor patrimonial. En este artículo se han examinado cada una de las partes del diseño, analizándolas bajo una visión histórica, compositiva y urbana. El aspecto más apreciable de la intervención de Nieto y Sobejano fue su versatilidad, ya que permitió que la rehabilitación arquitectónica del edificio admitiera integrar la Fundación de Arte y Pensamiento Martín Chirino convirtiendo el castillo en un elemento dinamizador del barrio y de Las Palmas, capaz de producir una regeneración urbana a partir de un proyecto arquitectónico-cultural.

A pesar de todas las vicisitudes sufridas por este destacado monumento y también de algunos de los cambios aplicados durante y al final de las obras que pudieron afectar a ciertos significados simbólicos enfatizados por los arquitectos, el resultado se puede considerar notable. Finalmente los habitantes de La Isleta y de toda la ciudad tienen que sentirse orgullosos de su fortaleza en cuanto representa un fundamental testigo de la historia y, gracias a la recualificación aquí descrita, de la modernidad. Se pasó de una visión, la de los vecinos de La Isleta, en la que su utilidad se reducía a ofrecer un servicio al barrio, a la más amplia de entender que un edificio de esta importancia pertenecía a todo el municipio, con un uso que prestigia a Gran Canaria y al archipiélago, demostrando una gran capacidad de integración en Las Palmas. La restauración integral del Castillo de La Luz es un hito notable en el contexto de la arquitectura defensiva de Canarias, además de poderse considerar un éxito de recuperación de una arquitectura pública que ha sido motor dinamizador del tejido cultural del barrio, la ciudad y la isla, con la acertada decisión de convertirla en sede de la Fundación de Arte y Pensamiento Martín Chirino, ya que la singular figura de este artista le otorga una dimensión de interés internacional. 
